\title{
Effects of Kaempferia parviflora extracts on reproductive parameters and spermatic blood flow in male rats
}

\author{
G Chaturapanich, S Chaiyakul, V Verawatnapakul and C Pholpramool \\ Department of Physiology, Faculty of Science, Mahidol University, Rama VI Road, Bangkok 10400, Thailand \\ Correspondence should be addressed to C Pholpramool; Email: sccpp@mahidol.ac.th
}

\begin{abstract}
Krachaidum (KD, Kaempferia parviflora Wall. Ex. Baker), a native plant of Southeast Asia, is traditionally used to enhance male sexual function. However, only few scientific data in support of this anecdote have been reported. The present study investigated the effects of feeding three different extracts of KD (alcohol, hexane, and water extracts) for 3-5 weeks on the reproductive organs, the aphrodisiac activity, fertility, sperm motility, and blood flow to the testis of male rats. Sexual performances (mount latency, mount frequency, ejaculatory latency, postejaculatory latency) and sperm motility were assessed by a video camera and computer-assisted sperm analysis respectively, while blood flow to the testis was measured by a directional pulsed Doppler flowmeter. The results showed that all extracts of KD had virtually no effect on the reproductive organ weights even after 5 weeks. However, administration of the alcohol extract at a dose of $70 \mathrm{mg} / \mathrm{kg}$ body weight (BW)/day for 4 weeks significantly decreased mount and ejaculatory latencies when compared with the control. By contrast, hexane and water extracts had no influence on any sexual behavior parameters. All types of extracts of KD had no effect on fertility or sperm motility. On the other hand, alcohol extract produced a significant increase in blood flow to the testis without affecting the heart rate and mean arterial blood pressure. In a separate study, an acute effect of alcohol extract of KD on blood flow to the testis was investigated. Intravenous injection of KD at doses of 10, 20 , and $40 \mathrm{mg} / \mathrm{kg}$ BW caused dose-dependent increases in blood flow to the testis. The results indicate that alcohol extract of KD had an aphrodisiac activity probably via a marked increase in blood flow to the testis.
\end{abstract}

Reproduction (2008) 136 515-522

\section{Introduction}

For several hundred years, people around the world have used locally grown plants as supplements to energize, vitalize, and eventually to improve male sexual functions. Up to now aphrodisiac activity of plant extracts including Eurycoma longifolia Jack (Ang et al. 2000), Terminalia catappa (Ratnasooriya \& Dharmasiri 2000), Lepidium meyenii (Maca; Zheng et al. 2000, Cicero et al. 2001), Tribulus terrestris (protodioscin; Gauthaman et al. 2002, 2003), Montanoa tomentosa (Carro- Juárez et al. 2004), and Fadogia agrestis (Yakubu et al. 2005) have been reported. The active components of these plants have not yet been identified. However, most investigators used aqueous or alcohol extracts that contain largely phenols, alkaloids, and saponins. Of these plants, the flavonoids, which are normally extracted by less polar or non-polar solvents, are not subjected to many studies, in spite of the fact that flavones and isoflavones are known to have estrogenic (Hiremath et al. 2000, Haddad \& Faqua 2001, Das et al. 2004) or androgenic activity (Qin et al. 2000, Yousef et al. 2005).

Krachaidum (KD, Kaempferia parviflora Wall. Ex. Baker), a native plant of Thailand, belongs to Zingiberaceae family. This plant has been used in Thai traditional medicine to treat various ailments such as inflammation, diarrhea, vertigo, and heart diseases. As a herbal product, KD has been used in different preparations such as fresh or dried rhizomes, dried powder in tea bags, and wine. It is usually claimed that KD increases male sexual activity and reproductive functions. However, the claim is based on consumer opinions. Scientific data in support of the aphrodisiac activity and reproductive functions are still controversial. Thus, Sudwan et al. $(2006,2007)$ have shown that alcohol extracts of KD and Boesenbergia rotunda (Krachai) had no effects on aphrodisiac activity and reproductive organs of male rats. Similarly, KD powder suspended in water exerted no androgen-like effects on reproductive organs of the castrated immature male rats (Trisomboon et al. 2007). On the other hand, Somphol et al. (2003) have shown that ground rhizome of Boesenbergia pandurata (former name of KD), increased ejaculation volume and had a tendency to increase total number, viability, and progressive motility of spermatozoa in rabbit semen. The positive effects of tea from KD on the seminal vesicle and spermatogenesis in rats have also been reported (jitjaingam et al. 2005). Such discrepancy is partly due to differences in the preparations and doses because chemical analyses of different fractions of KD 
extracts showed that hexane and alcohol extracts yielded phenols and flavonoids but at different percentages. Phenols are the major component of the alcohol extract while flavonoids are present mainly in the hexane extract. The water extract contains only small amounts of phenols and flavonoids (Sutthanut et al. 2007).

Although the effects of extracts of KD and its related plant on male reproductive organs and function have been reported, the mechanism by which these extracts mediate changes in reproductive function has not been elucidated. Since plants in the Zingiberaceae family such as curcuma and ginger have been shown to relax smooth muscles (Itthipanichpong et al. 2003) especially smooth muscle of blood vessels (Sasaki et al. 2003, Goto et al. 2005), it is possible that KD might relax vascular smooth muscle leading to an increase in blood flow to the reproductive organs and finally improvement of male reproductive functions. Therefore, the present study was designed to examine the effects of KD on aphrodisiac activity, reproductive functions, and spermatic blood flow in male rats.

\section{Results}

To investigate the long-term effect of KD on androgendependent and -independent organs, rats were treated with three different extracts for 5 weeks. We found that the relative weights of testes, epididymides, seminal vesicles, prostate glands, liver, kidneys, and levator ani muscle of KD-treated animals were not significantly different from the control group (Table 1).

To investigate the effect of KD on sexual behaviors, rats were treated with three extracts of KD as indicated in Experiment 1. Interestingly, the alcohol extract of KD significantly reduced both mounting and ejaculation latencies of the treated animals compared with those of the control group $(P<0.05)$ whereas similar effects were not observed with hexane or water extracts (Table 2). However, none of the alcohol, hexane, and water extracts of $\mathrm{KD}$ altered the percentage of fertility or sperm motility (Table 3 ).

The reduction in mounting and ejaculation latencies observed in the animals treated with the alcohol extract suggests an increase in sexual motivation that might be due to the favorable pharmacological effect of the extract on blood flow to male reproductive organs. To investigate this possibility, we measured spermatic blood flow after the sexual parameters were assessed. We found that chronic treatment of the alcohol extract of KD did not alter mean arterial blood pressure (mABP) or (resting) heart rate (HR). However, a significant increase $(P<0.05)$ in blood flow through the spermatic artery from $0.59 \pm 0.01 \mathrm{ml} / \mathrm{min}$ in control animals to $0.96 \pm 0.08 \mathrm{ml} / \mathrm{min}$ in $\mathrm{KD}$-treated animals was observed (Fig. 1).

It was of interest whether KD would produce an immediate effect on $\mathrm{mABP}$ and spermatic blood flow following an i.v. injection. Therefore, $\mathrm{mABP}$ and spermatic blood flow were measured after i.v. injections of the alcohol extract of KD at doses of 10, 20, and $40 \mathrm{mg} / \mathrm{kg}$ body weight (BW). Acetylcholine at a dose of $10 \mu \mathrm{g} / \mathrm{kg}$ BW was also administered as a positive control. As shown in Figs 2 and 3, all doses of alcohol extract, immediately produced dose-dependent decreases in $\mathrm{mABP}$. Meanwhile, dose-dependent increases in spermatic blood flow were observed after KD administration. Notably, acetylcholine significantly reduced $\mathrm{mABP}$ and increased spermatic blood flow in the same range as that of alcoholic extract at a dose of $20 \mathrm{mg} / \mathrm{kg}$ BW.

\section{Discussion}

In the present study, we have shown that among the three different fractions of KD extracts, only the alcohol extract has an effect on sexual behavior by shortening mount latency $(\mathrm{ML})$ and ejaculation latency (EL), which are considered to be the indicators of an increase in sexual motivation. However, there were no changes in other sexual parameters including mount frequency (MF), post-ejaculation latency (PEL), and $\mathrm{MF}_{30}$. Concurrent with the enhancement of sexual behaviors, spermatic blood flow was significantly augmented after treatment with the alcohol extract. All extracts had virtually no effect on androgen-dependent and -independent organs. Previous work has revealed flavones in the hexane extract of KD, all of which are methoxyflavones (Sutthanut et al. 2007). The finding in this study that hexane extract, which contains primarily flavones, had virtually no effect on sexual performance and reproductive organs of male rats suggests that methoxyflavones at the dose $70 \mathrm{mg} / \mathrm{kg}$ have neither androgenic nor estrogenic activity. Since flavones and isoflavones are phytoestrogens and hence have estrogenic or

Table 1 Effects of Krachaidum (KD) at a concentration of $70 \mathrm{mg} / \mathrm{kg}$ BW/day for 5 weeks on \% organ weight/BW.

\begin{tabular}{|c|c|c|c|c|c|c|c|}
\hline Group & Testis & Epididymis & Liver & Kidney & Seminal & Prostate & Levator Ani muscle \\
\hline $\mathrm{AC}$ & $0.98 \pm 0.02$ & $0.14 \pm 0.00$ & $3.86 \pm 0.18$ & $0.75 \pm 0.02$ & $0.17 \pm 0.01$ & $0.11 \pm 0.01$ & $0.26 \pm 0.01$ \\
\hline AKD & $1.01 \pm 0.03$ & $0.15 \pm 0.01$ & $3.78 \pm 0.09$ & $0.76 \pm 3.36$ & $0.16 \pm 0.02$ & $0.11 \pm 0.01$ & $0.25 \pm 0.01$ \\
\hline $\mathrm{HC}$ & $0.95 \pm 0.05$ & $0.14 \pm 0.01$ & $3.59 \pm 0.11$ & $0.74 \pm 0.02$ & $0.19 \pm 0.01$ & $0.09 \pm 0.00$ & $0.25 \pm 0.01$ \\
\hline HKD & $0.95 \pm 0.01$ & $0.14 \pm 0.00$ & $4.04 \pm 0.21$ & $0.78 \pm 0.02$ & $0.18 \pm 0.01$ & $0.09 \pm 0.01$ & $0.25 \pm 0.01$ \\
\hline WC & $0.98 \pm 0.03$ & $0.14 \pm 0.01$ & $3.80 \pm 0.06$ & $0.74 \pm 0.02$ & $0.18 \pm 0.01$ & $0.11 \pm 0.00$ & $0.24 \pm 0.01$ \\
\hline WKD & $0.93 \pm 0.01$ & $0.14 \pm 0.00$ & $4.10 \pm 0.08$ & $0.79 \pm 0.01$ & $0.17 \pm 0.01$ & $0.09 \pm 0.11$ & $0.25 \pm 0.00$ \\
\hline
\end{tabular}

$\mathrm{AC}$, alcohol control group; $\mathrm{AKD}$, alcohol extract of KD treatment group; $\mathrm{HC}$, hexane control group; HKD, hexane extract of KD treatment group; WC, water control group; WKD, water extract of KD treatment group. All values are shown in mean \pm s.E.M. $(n=8$ rats). 
Table 2 Effects of alcohol, hexane, and water extracts of Krachaidum (KD) at a dose of $70 \mathrm{mg} / \mathrm{kg}$ BW/day for 4 weeks on sexual behaviors.

\begin{tabular}{lccrrr}
\hline Group & ML $(\mathrm{min})$ & $\mathbf{M F}$ & $\mathbf{E L}(\mathrm{min})$ & $\mathbf{P E L}_{(\mathrm{min})}$ & $\mathbf{M F}_{\mathbf{3 0}}$ \\
\hline AC & $0.78 \pm 0.38$ & $44.75 \pm 7.75$ & $25.53 \pm 3.27$ & $7.78 \pm 0.77$ & $55.13 \pm 7.10$ \\
AKD & $0.18 \pm 0.12^{*}$ & $25.63 \pm 5.20$ & $8.95 \pm 2.80^{*}$ & $6.42 \pm 0.23$ & $53.50 \pm 6.13$ \\
HC & $0.61 \pm 0.19$ & $42.25 \pm 7.16$ & $17.00 \pm 2.69$ & $6.74 \pm 0.44$ & $62.50 \pm 5.47$ \\
HKD & $0.21 \pm 0.12$ & $41.00 \pm 8.59$ & $11.51 \pm 1.87$ & $6.96 \pm 0.41$ & $65.50 \pm 6.01$ \\
WC & $0.27 \pm 0.13$ & $28.38 \pm 5.32$ & $13.54 \pm 2.41$ & $8.19 \pm 0.73$ & $45.13 \pm 5.93$ \\
WKD & $0.13 \pm 0.15$ & $31.50 \pm 4.65$ & $12.21 \pm 2.22$ & $6.48 \pm 0.44$ \\
\hline
\end{tabular}

$\mathrm{AC}$, alcohol control group; $\mathrm{AKD}$, alcohol extract of $\mathrm{KD}$ treatment group; $\mathrm{HC}$, hexane control group; $\mathrm{HKD}$, hexane extract of $\mathrm{KD}$ treatment group; WC, water control group; WKD, water extract of KD treatment group. ML, mount latency; MF, mount frequency; EL, ejaculation latency; PEL, post-ejaculation latency; $\mathrm{MF}_{30}$, number of mounts within $30 \mathrm{~min}$. All values are shown in mean \pm S.E.M. $\left(n=8\right.$ rats). ${ }^{*} P<0.05$ significantly different from $\mathrm{AC}$.

anti-estrogenic activity (Haddad \& Fuqua 2001), it appears that methylation of the phenolic groups abolishes this activity. Although hexane and alcohol extracts yield similar flavones, the major components in each fraction are different. Thus, the three major flavones in the hexane extract are in the following order: $5,7-$ dimethoxyflavone $>5$-hydroxy, $-7,4^{\prime}$-dimethoxy-flavone $>5,7,4^{\prime}$-trimethoxyflavone. By contrast, those in the alcohol extract are 5,7, $4^{\prime}$-trimethoxyflavone $>5,7$ dimethoxyflavone $>3,5,7,3^{\prime}, 4$-pentamethoxyflavone. Furthermore, alcoholic extract contains a substantial amount of phenols while only a trace of these compounds is present in the hexane extract. On the other hand, aqueous extract has only small amount of flavones and no phenols (Sripanidkulchai et al. 2006). The differences in the composition of various extracts could explain the disparity of the results in this study.

As a Thai tonic herb for men, KD is usually consumed as tea or, more popularly, alcoholic drinks. However, most investigations of KD used aqueous extract. Tea prepared from KD powder at a dose of $120 \mathrm{mg} / \mathrm{kg}$ increased seminal vesicle weight, sperm density in the cauda epididymidis, and the diameter of seminiferous tubules of rats (Jitjaingam et al. 2005). On the other hand, aqueous suspension of KD powder at a dose of $1000 \mathrm{mg} / \mathrm{kg}$ failed to have any effect on the reproductive organs of the castrated immature rats although serum testosterone was elevated (Trisomboon et al. 2007). To our knowledge, only one study reported the effect of alcohol extract of KD on sexual behavior of male rats

Table 3 Effects of alcohol, hexane, and water extracts of Krachaidum (KD) at a dose of $70 \mathrm{mg} / \mathrm{kg}$ BW/day for 3 and 5 weeks on fertility and sperm motility.

\begin{tabular}{lccc}
\hline Group & ${\text { \% } \text { Fertility }^{\text {a }}}$ & ${\text { \% } \text { Motility }^{\text {b }}}$ & \% Progressive motility $^{\text {b }}$ \\
\hline AC & $72.5 \pm 7.6$ & $83.4 \pm 2.3$ & $37.4 \pm 2.6$ \\
AKD & $80.4 \pm 4.9$ & $81.2 \pm 2.2$ & $34.3 \pm 2.7$ \\
HC & $71.2 \pm 4.8$ & $81.1 \pm 3.1$ & $33.5 \pm 2.2$ \\
HKD & $73.6 \pm 6.3$ & $82.9 \pm 4.6$ & $34.3 \pm 2.3$ \\
WC & $74.9 \pm 5.4$ & $86.9 \pm 2.4$ & $37.0 \pm 2.1$ \\
WKD & $76.1 \pm 4.8$ & $89.5 \pm 1.5$ & $41.1 \pm 1.6$ \\
\hline
\end{tabular}

AC, alcohol control group; AKD, alcohol extract of KD treatment group; $\mathrm{HC}$, hexane control group; HKD, hexane extract of KD treatment group; WC, water control group; WKD, water extract of KD treatment group. All values are shown in mean \pm S.E.M., $n=8$ rats. ${ }^{\mathrm{a}}$ After 3 weeks KD treatment. ${ }^{\mathrm{b}}$ After 5 weeks KD treatment.
(Sudwan et al. 2006). However, these authors showed that KD (50\% alcohol extract) had virtually no effect on all sexual behavior parameters even though the dose was as high as $240 \mathrm{mg} / \mathrm{kg}$ and the duration was 60 days. The discrepancy between this and our study may be due to the active constituents in the extract and the animal model. In the present study, we used sexually experienced male rats. Our data, therefore, showed higher sexual activity compared with those of Sudwan et al. (2006). Indeed, their data indicated that the time of courtship significantly decreased over 30-min period even in the control group. In addition, we also used sexually experienced females for testing the mating behavior of the males. This might partly explain the relatively high values of $\mathrm{ML}, \mathrm{MF}$, and $\mathrm{EL}$ in the alcohol control group compared with other control groups, since the same females were used in testing the alcohol control and subsequently other groups. Taken together, our animal study provides some scientific support to the anecdotal belief that KD extract served as an alcoholic drink enhances male sexual libido. Other preparations such as tea fail to have such an effect. However, more studies in support of this notion are still required.

In order to investigate the mechanism by which KD enhanced sexual behavior, we measured blood flow to the testis via spermatic artery. Indeed, spermatic blood flow was markedly enhanced after treatment with alcohol extract of KD. The increase in blood flow to the testis would stimulate testosterone production and secretion which, in turn, acts on the central nervous system (CNS) and gonadal tissues to modulate male sexual behavior. There is evidence that testosterone production and secretion are closely related with the testicular blood flow (Damber \& Janson 1978). It has been shown that exposure of the rat testes to $\gamma$-irradiation caused a reduction in testis weight and disruption of spermatogenesis. Testicular blood flow per testis was decreased in proportion to the reduction in testicular weight and, as a result, the production of testosterone was markedly reduced (Wang et al. 1983). Furthermore, the measurement of testicular capillary blood flow in adult foxes by the radioactive inert gas clearance technique demonstrated that, during the mating period, at which time spermatozoa were observed in the testes and the testicular weight and 

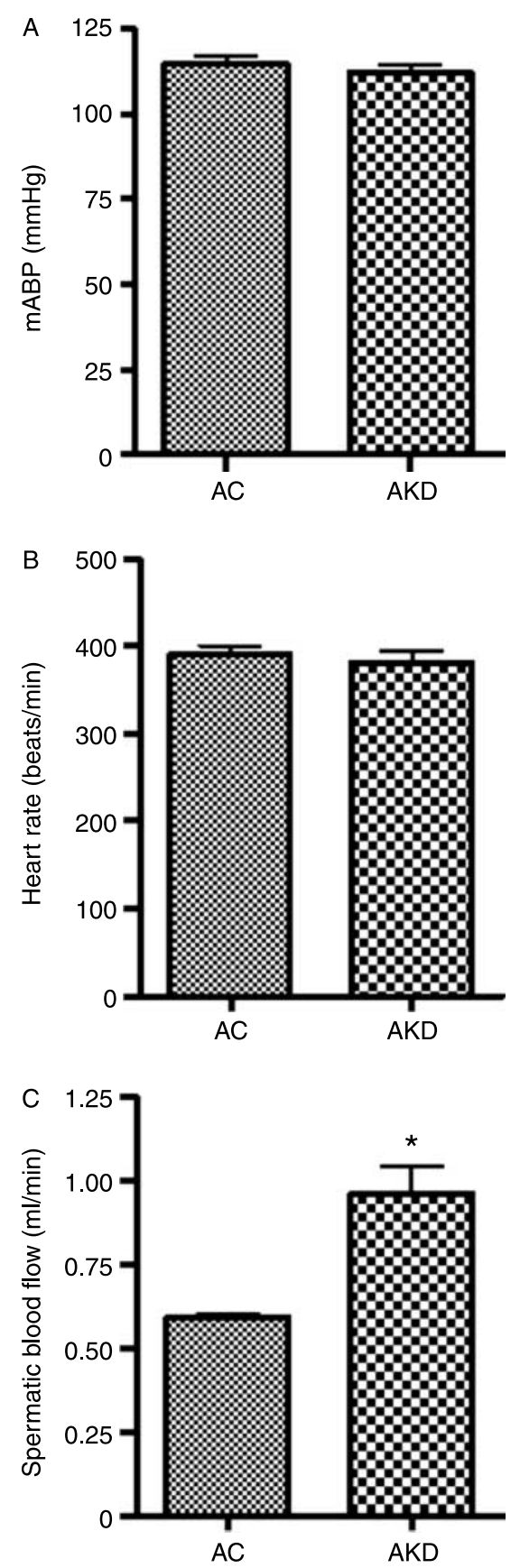

Figure 1 Effects of alcohol extract of Krachaidum (KD) treatment for 4 weeks on (A) mean arterial blood pressure, (B) heart rate, (C) spermatic blood flow. Values are mean \pm s.E.M. from eight rats. AC, alcohol control group; AKD, alcohol extract of KD treatment group; ${ }^{*} P<0.05$ significantly different from AC group.

circulating testosterone level were increased, testicular capillary blood flow increased (Joffre 1977). In contrast, during the period of testicular regression, testicular capillary blood flow and also androgenic activity decreased (Joffre 1977). However, the data in the present study do not support the notion that enhancement of sexual behaviors is due to the increase in testosterone production or secretion as a result of increased spermatic
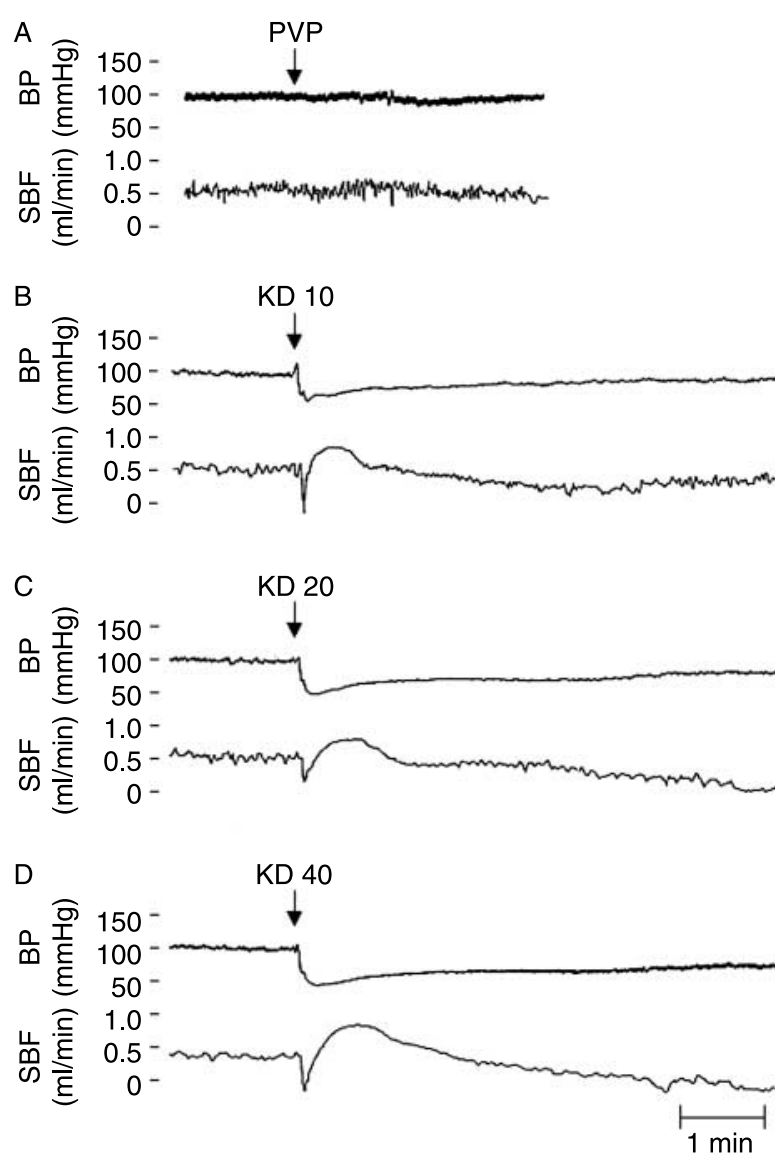

Figure 2 Representative tracings of the blood pressure (BP) and spermatic blood flow (SBF) in response to i.v. injections of $(A)$ vehicle (PVP) and alcohol extract of Krachaidum (KD) at doses of (B) 10, (C) 20, and (D) $40 \mathrm{mg} / \mathrm{kg} \mathrm{BW.}$

blood flow for several reasons. First, KD did not affect the weights of reproductive organs, such as epididymis, seminal vesicles and prostate glands, or the levator ani muscle, all of which are androgen-dependent tissues. Secondly, it has been reported that KD has no testosteronelike effect on the reproductive organs including seminal vesicles plus coagulating glands and ventral prostate and also the non-reproductive tissue such as levator ani muscle in the castrated immature rats treated with KD (Trisomboon et al. 2007). Thirdly, there is evidence that the aphrodisiac and fertility-enhancing properties of L. meyenii in healthy men have no correlation with serum reproductive hormone including testosterone (Gonzales et al. 2001, 2003). However, in view of the recent findings that in the castrated immature rats orally treated with dry KD powder in water for 5 days significantly increase serum testosterone levels were observed (Trisomboon et al. 2007), the possibility that KD enhances sexual behaviors via an increase in the testosterone cannot be ruled out.

Blood flow to the testis measured in the present study was in the same range as previously reported in which the flow was measured by various techniques in a variety of animal species during resting conditions, 


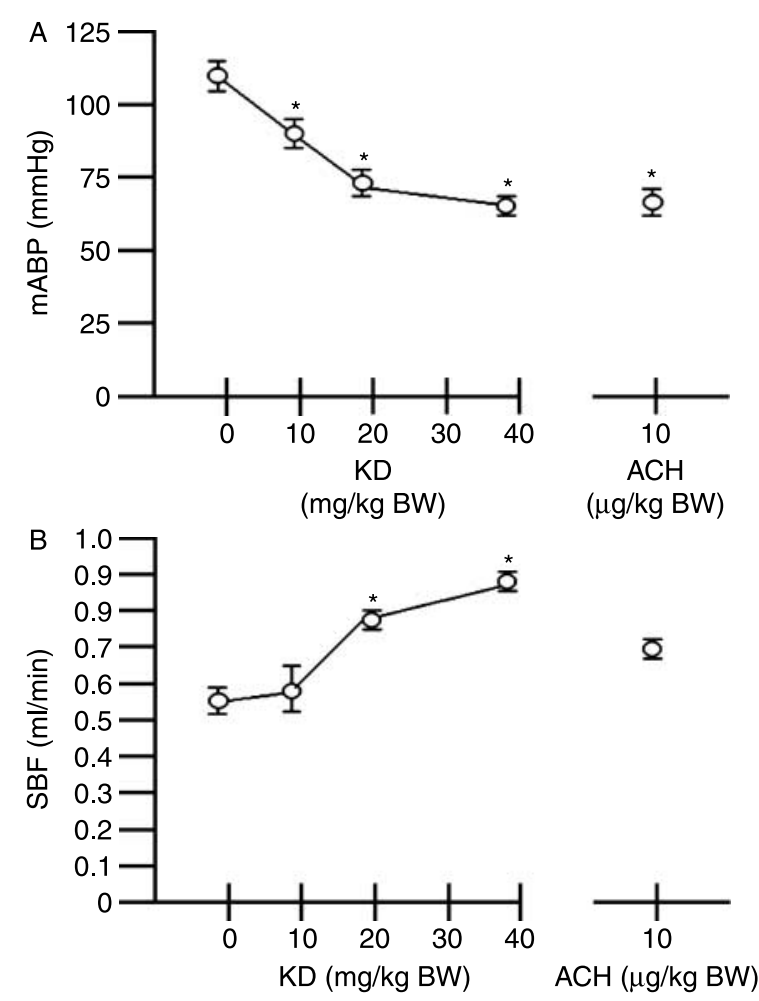

Figure 3 Effects of i.v. injections of alcohol extract of Krachaidum (KD) at doses of 10, 20, and $40 \mathrm{mg} / \mathrm{kg}$ BW on (A) mABP and (B) blood flow compared with acetylcholine $(\mathrm{ACH})$ at a dose of $10 \mu \mathrm{g} / \mathrm{kg} \mathrm{BW}$ in male rats. Values are mean \pm S.E.M. from eight rats. $* P<0.05$ significantly different from baseline value.

and when stimulated by vasodilators such as tropic hormone (Setchell et al. 1988) and human chorionic gonadotropins (Wang et al. 1984). The findings in the present study that an oral administration of alcohol extract of KD at a dose of $70 \mathrm{mg} / \mathrm{kg} \mathrm{BW} /$ day for 4 weeks significantly increased spermatic blood flow without changing the HR and the mean arterial blood pressure suggest that KD induces a local vasodilating effect. This result is consistent with the previous reports in humans and animals that plant extracts including those from garlic and flavonoid-rich cocoa induced vasodilation without altering the mean arterial blood pressure and HR (Naomi et al. 2003, Anim-Nyame et al. 2004). In order to confirm the vasodilating effect of KD, we performed an acute experiment in which alcohol extract of KD was intravenously injected at various doses. The results showed that KD caused a dose-dependent increase in blood flow to the testis, which was maintained for $30-100 \mathrm{~s}$. This effect resembled that after $10 \mu \mathrm{g} / \mathrm{kg}$ acetylcholine. The vasodilating response to KD could be a result of pharmacological effects of flavonoids, derivatives of methoxyflavones isolated from rhizome of KD (Sutthanut et al. 2007) on endothelial function. Studies in cultured human umbilical vein endothelial cells showed that KD produced a dose-dependent increase in nitrite concentration. Furthermore, expression of NOS mRNAs, the indicators of NO-cGMP induced vasodilation, was also enhanced (Wattanapitayakul et al. 2007). Studies in laboratory animals have shown that ginseng saponin enhanced libido and copulatory performance by directly acting on the CNS and gonadal tissues (Murphy \& Lee 2002). There is evidence that ginseng saponin facilitated penile erection by directly inducing the vasodilatation and relaxation of penile corpus cavernosum via nitric oxide mechanism (Chen \& Lee 1995). Moreover, alteration of the activity of hypothalamic cathecolamines was involved in the facilitation of copulatory behavior (Murphy et al. 1998). Whether K. parviflora promotes sexual behavior via acting centrally or peripherally needs further investigation.

In the present study, the reproductive functions, which are male fertility and sperm motility, were also investigated following administration of KD. The results showed that feeding the intact rats with a dose of $70 \mathrm{mg} / \mathrm{kg}$ BW/day of alcohol, hexane, and water extracts for 3 and 5 weeks had no effect on fertility nor sperm motility respectively. The failure to demonstrate the enhancing effect of KD may be due to the fact that these parameters are already high in the control animals. It has been shown that L. meyenii $(1500$ and $300 \mathrm{mg} / \mathrm{kg}$ per day for 4 months) improved sperm production and sperm motility in adult normal men (Gonzales et al. 2001). Whether sperm production is enhanced by KD is not known. By contrast, an administration of $500 \mathrm{mg} / \mathrm{kg}$ dose of Artocarpus heterophyllus seed suspension in 1\% methylcellulose in rats, failed to alter male fertility (Ratnasooriya $\&$ Jayakody 2002). The results obtained from the previous and present studies indicate that plant extracts produce a wide range of effect on sexual behavior parameters and reproductive functions. This, perhaps, might be explained by the differences in the active constituents of the plant, doses, and duration of treatment, and also experimental animal models used in the studies.

\section{Materials and Methods}

\section{Animals}

Male and female Sprague-Dawley rats, 5 weeks old and weighing 140-150 g, were obtained from the National Laboratory Animal Center, Mahidol University. They were housed separately in stainless steel hanging cages at $25^{\circ} \mathrm{C}$ and under a 12-h darkness: 12 -h light regimen with free access to food and water. At the time of experiment, the male (320$400 \mathrm{~g})$ and female $(200-250 \mathrm{~g})$ rats were sexually mature. All experiments were carried out according to the International Guiding Principles for Biomedical Research Involving Animals of CIOMS and approved by the Faculty of Science, Mahidol University Animal Care and Use Committee.

\section{Plant extracts}

KD rhizomes were collected from Loei province, Thailand. The alcohol, hexane, and water extracts of KD were provided by 
Assoc. Prof. Bungorn Sripanidkulchai, Director of Center for Research and Development of Herbal Health Product, Khon Kaen University, Thailand. The dried plant rhizome powder was prepared by the continuous Soxhlet extraction with solvents of different polarity in the following order: hexane, $95 \%$ ethanol, and water. After complete evaporation of the solvents, the dried extracts were used for gavage. Suspension of alcohol, hexane, and water extracts were prepared in $6.5 \%$ polyvinylpyrrolidone (PVP) in water, 5\% PVP dissolved in corn oil, and water respectively.

\section{Chemicals}

Estrogen ( $\beta$-estradiol 3-benzoate), progesterone (4-pregnene3,20-dione), and other reagent grade chemicals were purchased from Sigma. Sodium pentobarbital (Nembutal) was obtained from Sanofi (Sanofi Sante Animale SA, Paris, France).

\section{Sexual behavior study}

The sexual behaviors of male rats with proven fertility were tested with sexually experienced, ovariectomized female rats during the night (from 0630 to $1000 \mathrm{~h}$ ) in a quiet dark room at $22{ }^{\circ} \mathrm{C}$. Male rats were introduced into a rectangular aluminum observation cage $(28 \times 58 \times 17 \mathrm{~cm})$ with a wire mesh front and allowed to acclimatization for about $10 \mathrm{~min}$. Thereafter, a stimulus-receptive female rat was introduced and sexual behavior was recorded by a digital video camera recorder (DCR-TRV40E) for $30 \mathrm{~min}$. Female receptivity was induced by the sequential s.c. administration of $10 \mathrm{mg} / 100 \mathrm{~g}$ BW estradiol benzoate (Sigma Chemical Company) and $500 \mathrm{mg} / 100 \mathrm{~g} \mathrm{BW}$ progesterone (Sigma Chemical Company) for 48 and $4 \mathrm{~h}$ before the sexual behavior study respectively. Only receptive females exhibiting good lordosis in response to mounting when screened with non-experimental mature males were chosen in the study. The following sexual behavior parameters were registered according to the standard procedure (Gauthaman et al. 2002).

1) ML: the time interval ( $\mathrm{min}$ ) between the introduction of female into the cage until the first mount.

2) MF: the number of mounts from the first mount until ejaculation.

3) EL: the time ( $\mathrm{min})$ taken from the introduction of female into the cage until ejaculation.

4) PEL: the time (min) taken from the first ejaculation until the next mount.

5) $\mathrm{MF}$ within $30 \mathrm{~min}\left(\mathrm{MF}_{30}\right)$ : the number of mounts within $30 \mathrm{~min}$.

For ethical reasons, a set of sexually experienced females was used throughout. However, the females were randomly assigned to mate with the males.

\section{Male fertility}

The fertility of male rats was assessed by natural mating. Each mature male rat was individually housed in a large cage with two virgin female rats for 5 days. Successful mating was verified by the presence of spermatozoa in the vaginal smears taken every morning. The female rats showing thick clumps of spermatozoa in the vaginal smear were separated for 8-10 days, and then euthanized with overdose of diethyl ether (Sigma) for confirmation of pregnancy. The numbers of fetuses and corpora lutea were determined by the use of a dissecting microscope. The fertility of the male rat was expressed as the percentage fertility, calculated from the ratio of the number of fetuses to the number of corpora lutea $\times 100$. When both female rats were impregnated by the same male, the average value was used.

\section{Sperm motility}

After completion of 4-week KD feeding, animals were euthanized by overdose of i.p. sodium pentobarbital. The epididymis was removed and trimmed of fat. Spermatozoa were obtained by puncturing the distal cauda epididymidis and the epididymal fluid was collected into a hematocrit tube (id 1.1-1.2 mm; Sherwood Medical, St Louis, MO, USA) for $0.25-0.3 \mathrm{~cm}(2.65 \mu \mathrm{l})$ and rapidly transferred to $1 \mathrm{ml}$ modified Tyrode's solution. After $10 \mathrm{~min}$ incubation, the solution containing spermatozoa was dropped into an analysis chamber (depth $100 \mu \mathrm{M}$, Art. No. SC 100-01-c, The Netherlands). The sperm motility was then assessed by Hamilton Thorne Integrated Visual Optical System at $35^{\circ} \mathrm{C}$ and the parameters measured were the percentage of motile sperm and the percentage of progressively motile sperm (Goyal et al. 2001).

\section{Measurement of blood flow}

The male rat was anesthetized with an i.p. injection of $60 \mathrm{mg} / \mathrm{kg}$ BW of pentobarbital sodium. The level of surgical anesthesia was maintained during the experiment by supplementary doses of pentobarbital sodium (10-20\% of the initial dose) given intravenously when necessary. A tracheostomy was performed by inserting a short polyethylene cannula (ClayAdam, PE-240) to prevent airway obstruction. The left femoral artery was exposed and cannulated with a polyethylene catheter (Clay-Adam, PE-50) containing heparinized saline (50 IU heparin/ml). mABP and HR were measured by connecting the polyethylene catheter to a Statham P23 AC pressure transducer and a Grass polygraph recorder. The left femoral vein was also cannulated with a polyethylene catheter (Clay-Adam, PE-50) containing normal saline for the administration of KD and other substances at a volume of $0.1 \mathrm{ml}$ each time. Body temperature of the rat was monitored by a rectal thermister probe and maintained at $\sim 37^{\circ} \mathrm{C}$ throughout the experimental period with an overhead lamp. Spermatic blood flow was measured using a directional pulse Doppler flowmeter (545C-4, the University of lowa). After the abdominal wall over the spermatic artery was open, the vessel was isolated from the surrounding tissues. The piezoelectric crystal probe was gently placed around the vessel at the point where the spermatic artery enters the testis. The maximal blood flow was obtained by adjusting the range control, while listening to the audio from the speaker and turning the control for the best sound and monitoring the highest 
peaks of the output, which is a fine adjustment for the center of the flow stream.

Instantaneous volume flow was calculated as follow:

$Q=E D^{2} / 0.2712 \cos A$

where $Q$ is instantaneous volume flow $(\mathrm{ml} / \mathrm{min}), E$ is mean output (volt), $D$ is lumen diameter of probe embed tube, and $A$ is angle between sound beam and blood velocity vector which was constantly set at $45^{\circ}$.

\section{Experimental protocol}

Experiment 1: effects of extracts of $K D$ on male reproductive parameters

The animals were divided into six groups, eight rats per group. The first group (AC) received 6.5\% PVP dissolved in water serving as control for alcohol extract. The second group (alcohol extract of KD treatment group) received alcohol extract of KD at a dose of $70 \mathrm{mg} / \mathrm{kg} \mathrm{BW/day.} \mathrm{The} \mathrm{third} \mathrm{group}$ (hexane control group) received 5\% PVP dissolved in corn oil serving as control for hexane extract. The fourth group (hexane extract of KD treatment group) received hexane extract of KD at a dose of $70 \mathrm{mg} / \mathrm{kg}$ BW/day. The fifth group (water control group) received distilled water serving as control for water extract. The sixth group (water extract of KD treatment group) received water extract of $K D$ at a dose of $70 \mathrm{mg} / \mathrm{kg} \mathrm{BW} /$ day. Animals were fed by gavage daily with KD extracts or their vehicles at $2 \mathrm{ml} / \mathrm{kg}$ BW/day. After 3 weeks of treatment, they were allowed to have sexual experience by mating with virgin females, so as to test for their fertility. These male rats were further treated until the fourth and fifth weeks at which time sexual behaviors and sperm motility study were performed.

\section{Experiment 2: effect of $K D$ on spermatic blood flow}

Experiment 2.1: chronic effects of alcohol extract of $K D$ on blood pressure, $H R$, and spermatic blood flow. The rats were divided into two groups, eight rats each. The first group (AC) received $6.5 \%$ PVP in water serving as control. The second group received alcohol extract of KD dissolved in 6.5\% PVP as in Experiment 1 at a dose of $70 \mathrm{mg} / \mathrm{kg}$ BW/day for 4 weeks. After completion of KD treatment, the rats were prepared for the measurements of blood pressure, HR, and spermatic blood flow as described above.

Experiment 2.2: acute effects of alcohol extract of KD on blood pressure and spermatic blood flow. This part of study was carried out in eight intact rats receiving no treatment. After a steady spermatic blood flow recording was established for about $10 \mathrm{~min}$, alcohol extract of KD dissolved in 6.5\% PVP as in Experiment 1 at a dose of $10 \mathrm{mg} / \mathrm{kg}$ BW was administered through the femoral vein and spermatic blood flow was recorded until the effect of KD subsided. This protocol was repeated using 20 and $40 \mathrm{mg} / \mathrm{kg}$ doses to obtain a doseresponse curve. Finally, $10 \mu \mathrm{g} / \mathrm{kg}$ acetylcholine was administered as a positive control.

\section{Statistical analysis}

All results were expressed as mean \pm s.E.M. Significant differences between groups were analyzed by using one-way ANOVA followed by the post hoc Tukey's test for $\%$ fertility, $\%$ organs weight/BW, and blood flow data. The sexual behavior parameters were analyzed by Kruskall-Wallis ANOVA followed by Mann-Whitney $U$ test. The differences between two groups were considered to be statistically significant, if the statistical probability ( $P$ value) was less than 0.05 .

\section{Declaration of interest}

The authors declare that there is no conflict of interest that could be perceived as prejudicing the impartiality of the research reported.

\section{Funding}

This work was supported by a research grant from the National Research Council of Thailand.

\section{Acknowledgements}

The authors are grateful to Dr B Sripanidkulchai, Director of Center for Research and Development of Herbal Health Products, Khon Kaen University, for providing Kaempferia parviflora extracts and Department of Gynaecology, Ramathibodi hospital for allowing us to use Hamilton Thorne Integrated Visual Optical System for evaluation of sperm motility and Dr A Wongkularf for her advice on sperm motility analysis. The authors also gratefully acknowledge Dr A Dinudom for giving useful comments on the manuscript, $\mathrm{P}$ Pariyavuth for preparing figures, and C Sapeeya for typing the manuscript.

\section{References}

Ang HH, Cheang HS \& Yusof APM 2000 Effect of Eurycoma longifolia Jack (Tongkat Ali) on the initiation of sexual performance of inexperienced castrated male rats. Experimental Animals 49 35-38.

Anim-Nyame N, Sooranna SR, Johnson MR, Gamble J \& Steer PJ 2004 Garlic supplementation increase peripheral blood flow: a role for interleukin-6? Journal of Nutritional Biochemistry 15 30-36.

Carro-Juárez M, Cervantes E, Cervantes-Méndez M \& Rodriguez-Manzo G 2004 Aphrodisiac properties of Montanoa tomentosa aqueous crude extract in male rats. Pharmacology, Biochemistry, and Behavior 78 129-134.

Chen X \& Lee TJF 1995 Ginsenosides-induced nitric oxide mediated relaxation of the rabbit carvenosum. British Journal of Pharmacology 115 15-18.

Cicero AFG, Bandieri E \& Arletti R 2001 Lipidium meyenii Wallp. Improves sexual behavior in male rats independently from its action on spontaneous locomotor activity. Journal of Ethnopharmacology 75 225-229.

Damber JE \& Janson PO 1978 Testicular blood flow and testosterone concentration in spermatic venous blood of anaesthetized rats. Journal of Reproduction and Fertility 52 265-269.

Das S, Parveen S, Kundra CK \& Pereira BMJ 2004 Reproduction in male rats is vulnerable to treatment with the flavonoid-rich seed extracts of Vitex negundo. Phytotherapy Research 18I 8-13.

Gauthaman K, Adaikan PG \& Prasad RNY 2002 Aphrodisiac properties of Tribulus Terrestris extract (Protodioscin) in normal and castrated rats. Life Sciences 71 1385-1396. 
Gauthaman K, Ganesan AP \& Prasad RN 2003 Sexual effects of puncturevine (Tribulus terrestris) extract (protodioscin): an evaluation using a rat model. Journal of Alternative and Complementary Medicine 9 257-265.

Gonzales GF, Cordova A, Gonzales C, Chung A, Vega K \& Villena A 2001 Lepidium meyenii (Maca) improved semen parameters in adult men. Asian Journal of Andrology 3 301-303.

Gonzales GF, Cordova A, Vega K, Chung A, Villena A \& Gonez C 2003 Effect of Lepidium meyenii (Maca), a root with aphrodisiac and fertilityenhancing properties, on serum reproductive hormone levels in adult healthy men. Journal of Endocrinology 176 163-168.

Goto H, Sasaki Y, Fushimi H, Shibahara N, Shimada Y \& Komatsu K 2005 Effect of curcuma herbs on vasomotion and hemorheology in spontaneously hypertensive rat. American Journal of Chinese Medicine 33 449-457.

Goyal HO, Braden TD, Mansour M, Williams CS, Kamaleldin A \& Srivastava KK 2001 Diethylstilbestrol-treated adult rats with altered epididymal sperm numbers and sperm motility parameters, but without alterations in sperm production and sperm morphology. Biology of Reproduction 64 927-934.

Haddad N \& Fuqua JS 2001 Phytoestrogens: effects on the reproductive system. Endocrinologist 11 498-505.

Hiremath SP, Badami S, Hunasagatta SK \& Patil SB 2000 Antifertility and hormonal properties of flavones of Striga orobanchioides. European Journal of Pharmacology 391 193-197.

Itthipanichpong C, Ruangrungsi N, Kemsri W \& Sawasdipanich A 2003 Antispasmotic effects of curcuminoids on isolated guinea-pig ileum and rat uterus. Journal of the Medical Association of Thailand $\mathbf{8 6}$ (Supplement 2) S299-S309.

Jitjaingam A, Kakaew A, Saenphet K, Saenphet S \& Aritajat S 2005 Effects of Kaempferia parviflora Wall. Ex. Baker on reproductive organs hematology and kidney function of male rats. Proceedings of the $31 \mathrm{st}$ Congress on Science and Technology of Thailand, Suranaree University of Technology, Nakhon Ratchasima, Thailand.

Joffre M 1977 Relationship between testicular blood flow, testosterone secretion and spermatogenic activity in young and adult wild red foxes (Vulpes vulpes). Journal of Reproduction and Fertility 51 35-40.

Murphy LL \& Lee TJF 2002 Ginseng, sex behavior and nitric oxide. Annals of the New York Academy of Sciences 962 372-377.

Murphy LL, Cadena RS, Chavez D \& Ferraro JS 1998 Effect of American ginseng Panax quinquefolium on male copulatory behavior in the rat. Physiology and Behavior 64 445-450.

Naomi DLF, Hughehs M, Herman MG \& Hollenberg NK 2003 Flavonoidrich cocoa induces nitric oxide dependent vasodilation in healthy humans. Journal of Hypertension 21 2281-2286.

Qin DN, She BR, She YC \& Wang JH 2000 Effects of flavonoids from semen cuscutae on the reproductive system in male rats. Asian Journal of Andrology 2 99-102.

Ratnasooriya WD \& Dharmasiri MG 2000 Effects of Terminalia catappa seeds on sexual behavior and fertility of male rats. Asian Journal of Andrology 2 213-219.

Ratnasooriya WD \& Jayakody JR 2002 Artocarpus heterophyllus seeds inhibit sexual competence but not fertility of male rats. Indian Journal of Experimental Biology 40 304-308.

Sasaki Y, Goto H, lohda C, Hatanaka F, Shibahara N, Shimada Y, Terasawa K \& Komatsu K 2003 Effect of curcuma drugs on vasomotion in isolated rat aorta. Biological \& Pharmaceutical Bulletin 26 1135-1143.
Setchell BP, Maddock S \& Brook DE 1988 Anatomy, vasculature, innervation and fluids of the male reproductive tract. In The Physiology of Reproduction, 2 edn, pp 1063-1175. Eds E Knobil \& LD Neill. New York: Raven Press.

Somphol N, Hongkhuntod P, Vongpralab T, Sanchaisuriya P, Chinchiyanont W \& Prasuk Y 2003 Effect of Boesenbergia pandurata (black rhizome) supplementation on semen characteristics in male rabbits. Proceedings of the Annual Academic Meeting of the Faculty of Agriculture, Khon Kaen University, Khon Kaen, Thailand, pp 46-54.

Sripanidkulchai B, Yenjai C, Sutthanut K \& Veerapreeyakul N 2006 Development of monograph, identification method, quantitative analysis method by using chromatography technique and biological activities testing of Kaempferia parviflora active ingredients. Proceedings of the Seminar on Development of Herbs for Industry, National Research Council of Thailand, Bangkok, Thailand, pp 194-215.

Sudwan P, Saenphet K, Saenphet S \& Suwansirikul S 2006 Effect of Kaempferia parviflora Wall. ex, Baker on sexual activity of male rats and its toxicity. Southeast Asian Journal of Tropical Medicine and Public Health 37 (Supplement 3) 210-215.

Sudwan P, Saenphet K, Aritajat S \& Sitasuwan N 2007 Effects of Boesenbergia rotunda (L.) Mansf. on sexual behavior of male rats. Asian Journal of Andrology 9 849-855.

Sutthanut K, Sripanidkulchai B, Yenjai C \& Jay M 2007 Simultaneous identification and quantitation of 11 flavonoid constituents in Kaempferia parviflora by gas chromatography. Journal of Chromatography. A $1143227-233$.

Trisomboon H, Watanabe G, Wetchasit P \& Taya K 2007 Effect of daily treatment with Thai Herb, Kaempferia parviflora, in Hershberger assay using castrated immature rats. Journal of Reproduction and Development 53 352-356.

Wang J, Galil KA \& Setchell BP 1983 Changes in testicular blood flow and testosterone production during aspermatogenesis after irradiation. Journal of Endocrinology 98 35-45.

Wang JM, Gu CH, Qian ZM \& Jing GU 1984 Effects of gossypol on testicular blood flow and testosterone production in rats. Journal of Reproduction and Fertility 70 127-133.

Wattanapitayakul SK, Suwatronnakorn M, Chularojmontri L, Herunsalee A, Niumsakul S, Charuchongkolwongse S \& Chansuvanich N 2007 Kaempferia parviflora ethanolic extract promoted nitric oxide production in human umbilical vein endothelial cells. Journal of Ethnopharmacology $110559-562$.

Yakubu MT, Akanji MA \& Oladiji AT 2005 Aphrodisiac potentials of the aqueous extract of Fadogia agrestis (Schweinf. Ex Hierr) on male albino rats. Asian Journal of Andrology 7 399-404.

Yousef MI, Esmail AM \& Baghdadi HH 2005 Effect of isoflavones on reproductive performance, testosterone levels, lipid peroxidation, and seminal plasma biochemisty of male rabbits. Journal of Environmental Science and Health. Part. B 39 819-833.

Zheng BL, He K, Rogers L, Shao Y, Huang ZY, Yan SI, Qien LC \& Zheng OY 2000 Effect of a lipidic extract from Lipidium meyenii on sexual behavior in mice and rats. Urology 55 598-602.

Received 13 February 2008

First decision 14 March 2008

Revised manuscript received 29 May 2008

Accepted 8 July 2008 